\title{
On-site Identification of Dynamic Annular Seal Forces in Turbo Machinery Using Active Magnetic Bearings - An Experimental Investigation
}

\author{
Lauridsen, Jonas S.; Santos, IImar F.
}

Published in:

Journal of Engineering for Gas Turbines and Power

Link to article, DOI:

$10.1115 / 1.4038755$

Publication date:

2018

Document Version

Peer reviewed version

Link back to DTU Orbit

Citation (APA):

Lauridsen, J. S., \& Santos, I. F. (2018). On-site Identification of Dynamic Annular Seal Forces in Turbo Machinery Using Active Magnetic Bearings - An Experimental Investigation. Journal of Engineering for Gas Turbines and Power, 140(8), [082501]. https://doi.org/10.1115/1.4038755

\section{General rights}

Copyright and moral rights for the publications made accessible in the public portal are retained by the authors and/or other copyright owners and it is a condition of accessing publications that users recognise and abide by the legal requirements associated with these rights.

- Users may download and print one copy of any publication from the public portal for the purpose of private study or research.

- You may not further distribute the material or use it for any profit-making activity or commercial gain

- You may freely distribute the URL identifying the publication in the public portal 


\title{
On-site Identification of Dynamic Annular Seal Forces in Turbo Machinery Using Active Magnetic Bearings - An Experimental Investigation
}

\author{
Jonas S. Lauridsen* \\ PhD student \\ Department of Mechanical Engineering \\ Technical University of Denmark \\ Email: jonlau@mek.dtu.dk \\ IImar F. Santos ${ }^{\dagger}$ \\ Professor \\ Department of Mechanical Engineering \\ Technical University of Denmark \\ Email: ifs@mek.dtu.dk
}

Significant dynamic forces can be generated by annular seals in rotordynamics and can under certain conditions destabilize the system leading to a machine failure. Mathematical modeling of dynamic seal forces are still challenging, especially for multiphase fluids and for seals with complex geometries. This results in much uncertainty in the estimation of the dynamic seal forces which often leads to unexpected system behavior.

This paper presents the results of a method suitable for on-site identification of uncertain dynamic annular seal forces in rotordynamic systems supported by Active Magnetic Bearings (AMB). An excitation current is applied through the AMBs to obtain perturbation forces and a system response, from which, the seal coefficients are extracted by utilizing optimization and a-priori information about the mathematical model structure and its known system dynamics. As a study case, the method is applied to a full-scale test-facility supported by two radial AMBs interacting with one annular center mounted test-seal. Specifically, the dynamic behavior of a smooth annular seal with high preswirl and large clearance (worn seal) is investigated in this study for different excitation frequencies and differential pressures across the seal. The seal coefficients are extracted and a global model on reduced state-space modal form are obtained using the identification process. The global model can be used to update the model based controller to improve the performance of the overall system. This could potentially be implemented in all rotordynamic systems supported by AMBs and sub-

\footnotetext{
*Address all correspondence related to ASME style format and figures to this author.

${ }^{\dagger}$ Address all correspondence related to ASME style format and figures to this author.
}

jected to seal forces or other fluid film forces.

\section{Introduction}

Bulkflow and Computational Fluid Dynamics (CFD) methods are typically used to obtain the static and dynamic properties of seals. The development of the bulkflow code goes back to the 1980 s and is still in development [1-7]. This technique usually assumes that the flow through the seals can be represented using one-dimensional partial differential equations coupled to the equation describing the rotor lateral motion. In many cases, as for example for complex seal geometries, these simplified 1D assumptions do not hold and parameter adjustments and corrections are needed. Thus, the bulkflow code is usually heavily linked to empirical parameters and lacks generality. The bulkflow codes are however widespread in industrial rotordynamic software such as ISOTSEAL and XLTRC. ISOTSEAL and XLTRC have been developed by Texas A\&M University in the USA for annular seals and for complete lateral and torsional rotordynamic analysis of rotating machinery including pumps, compressors and turbines, respectfully. The approach used by ISOTSEAL [1,8-11] is based on bulkflow theory to simplify the system from three-dimensional to one-dimensional. Two set of equations are considered, the zeroth-order equations for steady-state solutions (leakage and force) and the firstorder equations for dynamic solutions (stiffness and damping). The approach used by XLTRC for lateral rotordynamics is based on Timoshenko beam and finite-element formulation to model the rotor and housing. The bulkflow codes due to having a simple model structure are computationally inexpensive which, in that regard, makes them at- 
Copyright tractive compared to CFD. CFD models have on the other hand been shown to be able to find seal forces even with complex geometries without tuning empirical constants, which makes them more general than bulkflow codes [10]. CFD simulations can be extremely time demanding and computationally heavy, since full 3D flow and pressure fields have to be calculated in time. That said, CFD methods are becoming increasingly popular as computational power in computers rapidly increases [12].

Modeling of seal forces can be very challenging. This is made clear by a survey conducted in 2007 , where 20 survey participants from both industry and academia were asked to predict the dynamics of a gas labyrinth seal and consequently the rotordynamic behavior [13]. The seals dynamics was predicted using bulkflow and CFD methods. The survey showed large variations in the results and emphasizes the need for continuous efforts in modeling and uncertainty handling of seal forces, even for single phase flow condition, as presented in the article. Multiphase flows, i.e. where the fluid is a mixture of gas and liquid, add further complexity to the modeling and, consequently, higher uncertainties in the prediction of the seal dynamics are to be expected.

There are two usual ways of experimentally identifying dynamic force coefficients of seals: i) by keeping the lateral movements of the rotor constrained while shaking the seal housing or ii) by shaking the rotor laterally while keeping the seal housing constrained. Examples of method $i$ are more common and thoroughly documented in the literature with testing performed at university laboratories [14-16]. Examples of method ii where AMBs are used to identify and characterize fluid film forces are reported in [12,17-22]. The design of a full scale magnetic bearing test-facility to levitate and excite the rotor with an annular test-seal mounted in the center of the rotor is presented in [17]. This work also presents the identification results of dynamic force coefficients of a labyrinth seal for high pressure turbomachinery. The labyrinth seal forces are compared with CFD results in [12]. Identification of seal forces in a flexible rotor system using AMBs is shown in [22]. The AMB forces are measured using fiber-optic strain gauges that are bonded to the stator poles of the AMBs. Stiffness and damping coefficients are determined using a frequency domain identification method, utilizing an FE rotor model which is adjusted to match the characteristics of the test rotor in free-free conditions. The use of AMB for identification of fluid film forces of a journal bearing have been investigated among others in [18-21]. Here the forces are measured by use of hall sensors and the dynamics of the journal bearing is represented by mass, stiffness and damping matrices which are a function of the rotational speed and pocket pressure ratio.

This paper presents a force calibration free identification scheme for updating parameters in a global rotordynamic model with AMBs and seal forces. This method is applied to a test-facility and the results are shown. Thus, no force transducers or calibration equipment are needed to characterize magnetic forces like those suggested in [12, 17-19, 22]. The idea, though, is similar to the plausibility check, shown in [12], where the seal dynamics are identified as the residual dynamics between the global model and a baseline AMBrotor model, assuming the baseline model is a-priori known. Hence, the approach in [12] still relies on traditional modeling and calibration of the AMB-rotor system. The method, presented in this paper is a general way to identify all uncertain/unknown parameters of an AMB-rotor-seal system systematically without changing the model structure or model order. This is particular useful in applications in which the seal forces are hard to model in advance, like for example in subsea applications in which the fluid can be a mixture between gas and liquid. In these cases it would be extremely useful to identify the forces in-situ to prevent system failure by improved model capabilities or to update the controller of the AMBs to improve stability and performance.

\section{Experimental Facilities}

The experimental facilities used for this work consist of a AMB-based rotordynamic test bench with a seal house presented in Fig. 1 and a cross-section view in Fig. 2. Two AMBs radially support a symmetric rigid rotor which is driven by an asynchronous motor through an intermediate shaft and a flexible coupling, as seen in Fig. 3. Angular contact ball bearings, supporting the intermediate shaft housed in the intermediate shaft pedestal, compensate for axial forces acting on the rotor. The radial AMBs are of the eight pole heteropolar type, see Fig. 4.



Fig. 1. Test facility overview. (1) AMB A, (2) Seal house, (3) AMB B. Figure adapted from [23].

In Fig. 4 both the global reference frame denoted by $x, y$ and the actuator reference frame denoted by $\zeta, \eta$ are introduced. The actuators are tilted $45^{\circ}$ with respects to the global reference frame. Throughout the paper subscripts $\zeta, \eta$ are used to denote quantities belonging to the actuators aligned with the respective axes of the stator reference frame. The AMBs are supplied by four commercially available $3 \mathrm{~kW}$ switch-mode laboratory amplifiers. The AMBs are control- 


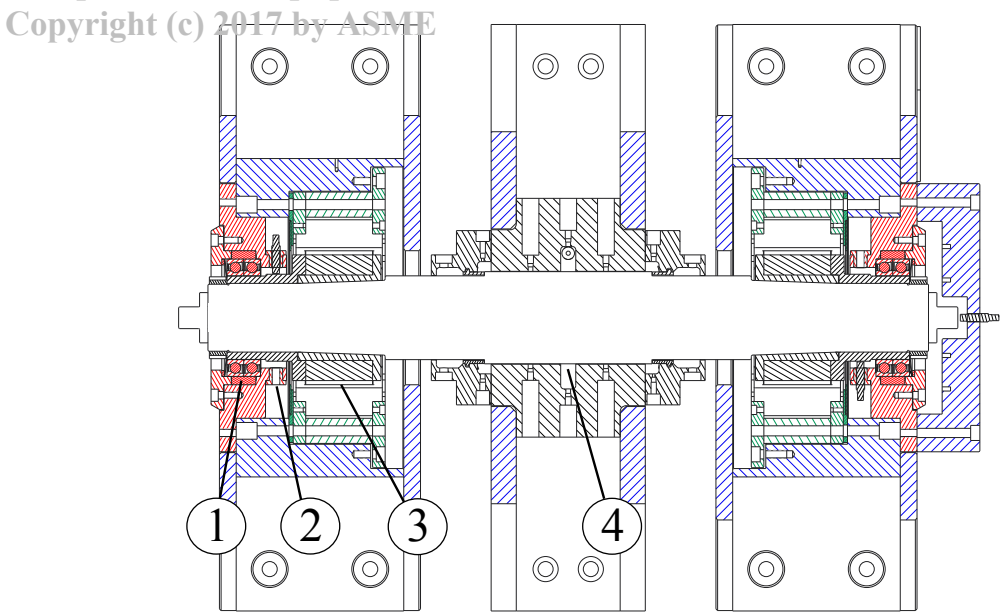

Fig. 2. Full section view of testrig with (1) backup bearing, (2) displacement sensor, (3) rotor and stator of the AMB, (4) seal house. Figure adapted from [23].

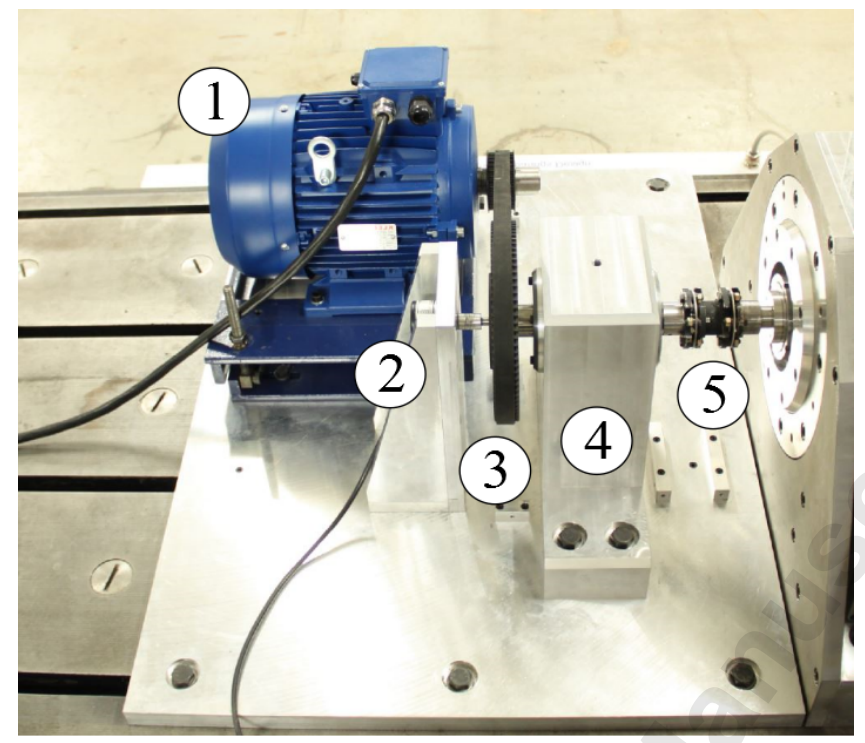

Fig. 3. Connecting the motor to the shaft. (1) motor, (2) Encoder, (3) Belt drive, (4), Intermediate shaft pedestal, (5) Flexible coupling. The figure is adapted from [23].

led using a standard decentralized PID scheme, which would typically be used in industrial applications. The rotor is kept in the center of the seal, which is slightly offset from the magnetic/effective center of the AMBs.

The smooth cylindrical seal, mounted in the center of the shaft, is installed as a back-to-back configuration, hence two symmetrical seals are placed with the idea to cancel out possible axial fluid film forces. The seal housing and its features are illustrated in Fig. 5. It is designed with primary and secondary discharge seals to avoid any liquid entering the AMBs, since the test facility is designed to operate with both gas, liquid and mixtures between gas and liquid. The fluid used in this article is gas (air), though. The fluid is injected by four highly angled nozzles to obtain a high preswirl



Fig. 4. Test bench AMB showing the AMB actuator and global reference frames. The figure is adapted from [24].

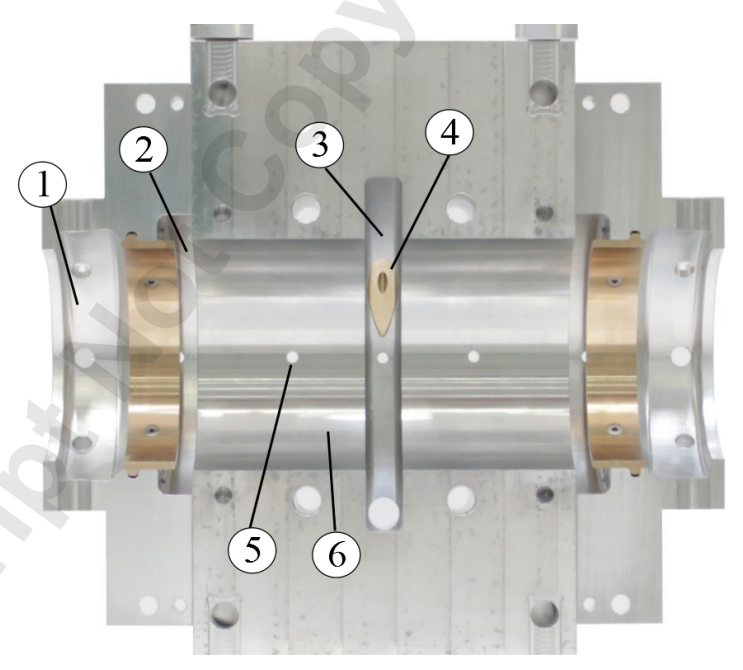

Fig. 5. Cross section of the seal house. (1) secondary discharge cavity, (2) discharge cavity, (3) inlet injection cavity, (4) inlet nozzle,(5) pressure sensor, (6) seal surface. The figure is adapted from [24].

ratio, hence the fluid is already rotated at the inlet of the seal. A cross section of the injection system is shown in Fig. 6.

A full description of the test facility can be found in [23], which also presents the calibration of the complete system. Design parameters for the rotordynamic test bench can be found in Table 1.

\section{Identification}

The idea is to establish a precise global mathematical model of the test-facility consisting of an updated nominal model representation. Hence, a nominal model is constructed using conventional modeling methods and where the uncertain or unknown coefficients are identified in-situ. Since the mathematical structure is kept intact and the identified parameters have physical meanings, this method is preferred over e.g blackbox modeling. The global model can for example be used to investigate the performance of the test- 


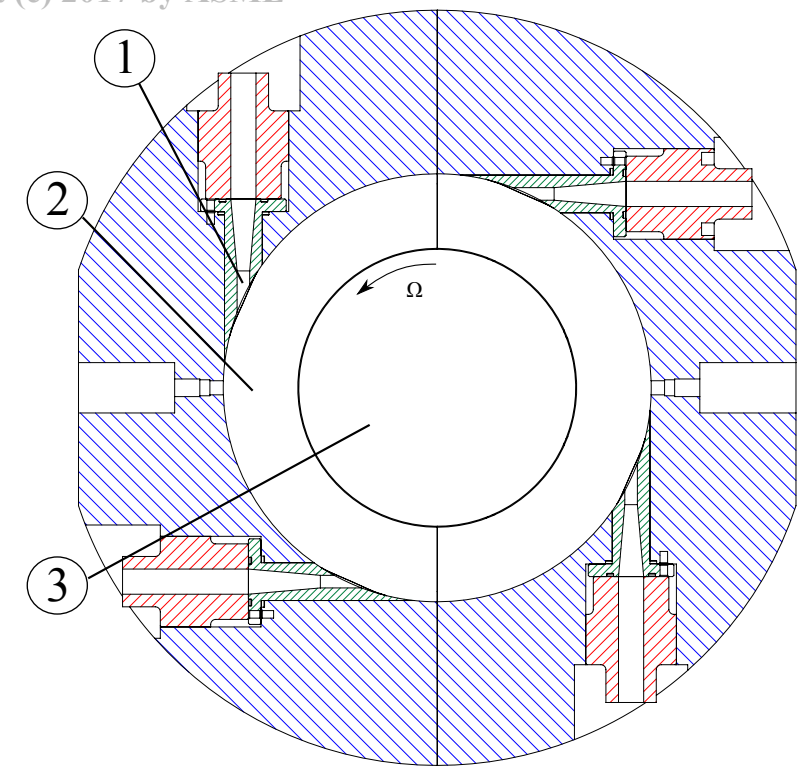

Fig. 6. Cross section of the seal house at the inlet cavity section. (1) inlet injection nozzle, (2) inlet cavity, (3) shaft. The figure is adapted from [24]

facility operating at other speeds or seal configurations than the one in-which the model was identified for. Also, analysis of robust stability and performance and the design of robust controllers can be carried out with respect to uncertain system parameters [25].

\subsection{Mathematical representation of the nominal AMB- rotor-seal system}

The nominal mathematical system representation of the test-facility consist of a finite element model of the rotor, dynamic model of AMB actuation forces, the coupling dynamics and the seal dynamics, described next.

\subsubsection{Model of AMB Forces}

The model of the magnetic bearing is simplified to describe the forces acting on the rotor as function of the rotor lateral displacements to AMB $s_{x}$ and the control current $i_{x}$. The linearized expression of the forces for a given direction

Table 1. Design parameters for the rotordynamic test bench

\begin{tabular}{lrl} 
Rotor length & 860 & $\mathrm{~mm}$ \\
Rotor assembly mass & 69 & $\mathrm{~kg}$ \\
1st rotor bending mode @ & 550 & $\mathrm{~Hz}$ \\
Stator inner diameter & 151 & $\mathrm{~mm}$ \\
Nominal radial air gap & 0.5 & $\mathrm{~mm}$ \\
Winding configuration & N-S-S-N-N-S-S-N & {$[-]$} \\
Number of windings & 36 & {$[-]$} \\
\hline
\end{tabular}

is given as [26]

$$
f_{b}\left(i_{x}, s_{x}\right)=K_{i} i_{x}+K_{s} s_{x}
$$

where $K_{i}$ are $K_{s}$ are constants. Initial estimates of $K_{i}$ and $K_{s}$ have been obtained using first principle methods. The dynamics of the electromechanical system, including the inductance of the coil and the amplifiers, is approximated as a second order system, denoted $G_{a c t}$

$$
G_{a c t}=\frac{\omega_{n}^{2}}{s^{2}+\zeta \omega_{n} s+\omega_{n}}
$$

where the damping coefficient and natural frequency are empirically found to be $\zeta=0.9$ and $\omega_{n}=1360 \mathrm{rad} / \mathrm{s}(216 \mathrm{~Hz})$.

\subsubsection{Model of Dynamic Seal Forces}

The dynamic seal forces are commonly described by their linearized force coefficients: stiffness, damping and sometimes mass matrices. Mass coefficients are hereby neglected since the fluid used is air [1]

$$
\left[\begin{array}{l}
f_{\zeta} \\
f_{\eta}
\end{array}\right]=\left[\begin{array}{cc}
K & k \\
-k & K
\end{array}\right]\left[\begin{array}{l}
\zeta \\
\eta
\end{array}\right]+\left[\begin{array}{cc}
C & c \\
-c & C
\end{array}\right]\left[\begin{array}{c}
\dot{\zeta} \\
\dot{\eta}
\end{array}\right]
$$

This model has a symmetric structure since the shaft is assumed to be in the center of the seal. The coefficients are generally a function of the rotational speed and the excitation frequency.

\subsubsection{Model of Flexible Coupling Forces}

The force of the flexible coupling, as shown in Fig. 3, is considered as a direct stiffness matrix for radial movements. Torsional stiffness is considered small and is neglected for simplicity.

$$
\left[\begin{array}{l}
f_{\zeta} \\
f_{\eta}
\end{array}\right]=\left[\begin{array}{cc}
K_{c_{\zeta}} & 0 \\
0 & K_{c_{\eta}}
\end{array}\right]\left[\begin{array}{l}
\zeta \\
\eta
\end{array}\right]
$$

\subsubsection{Model of Shaft}

The dynamic behavior of the rotating shaft is mathematically described using the Finite Element (FE) method and Bernoulli-Euler beam theory considering the gyroscopic effects of the shaft and discs [27]. The shaft model is built using 40 node points with 4 degrees of freedom each, i.e. $x$ and $y$ direction, and the rotation around the $x$ and $y$ axes. It yields 320 states in total. The rotational speed is kept at zero for this study to isolate the seal effects. The tangential fluid flow is induced by means of four injectors built as illustrated in Fig. 6, what leads to high preswhirl effect. The global rotordynamic system $G_{f}$ consisting of the finite element model of the shaft, negative stiffness forces from the AMB, the 
Copyright (ctifinness and damping of the seal and the stiffness of the coupling can be written in state space form as

$$
\dot{x}_{f}=A_{f} x_{f}+B_{f} u, \quad y=C_{f} x_{f}
$$

Using modal truncation techniques, real left and right modal transformation matrices are obtained which transform the full order FE system to a reduced form

$$
x=T_{L}^{T} x_{f}, \quad A=T_{L}^{T} A_{f} T_{R}, \quad B=T_{L}^{T} B_{f}, \quad C=C_{f} T_{R}
$$

The first bending mode of the shaft lies at approximately $550 \mathrm{~Hz}$. Since this is substantially above the frequency range of interest in this work, the shafts is assumed rigid and all bending modes have thus been removed in the reduced order model. The FE model is selected though for generality and for possibility of extending the model to include some of the bending modes if needed.

\subsection{Changed Plant Representation}

The nominal rotordynamic model consists of the reduced order shaft model, the negative stiffness from the AMBs and the nominal stiffness and damping from the seals. The updated model $G_{f i}$ is constructed using the nominal model and the change of dynamics representation, which are combined and written in Linear Fractional Transformation (LFT) form, illustrated in Fig. 7 [28]. Here $\theta$ is a diagonal matrix

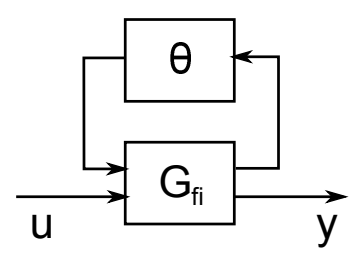

Fig. 7. Updated/changed plant representation using upper LFT, $G_{\text {updated }}=\mathcal{F}_{u}\left(G_{f i}, \theta\right)$

representing the normalized parameters to be identified. $G_{f i}$ can be written in state space form, as shown in Eq. (7), where $\mathrm{A}, \mathrm{B}$ and $\mathrm{C}$ are the nominal system matrices. Here the input and output matrices are extended from the nominal model to include the input and output mapping $B_{\theta}$ and $C_{\theta}$. Note that no extra system dynamics is added since the LFT only changes the nominal system matrix A.

$$
G_{f i}=\left[\begin{array}{c|cc}
A & B_{\theta} & B \\
\hline C_{\theta} & 0 & 0 \\
C & 0 & 0
\end{array}\right]
$$

$B_{\theta}$ and $C_{\theta}$ are selected to represent changes in the nominal plant caused by AMB bias stiffness, mechanical stiffness from coupling, stiffness and damping from the seal. The structures of e.g. $B_{\theta}$ and $\theta$ are given as

$$
B_{\theta}=\left[B_{\theta K_{s}}, B_{\theta K_{\text {coupling }}}, B_{\theta K_{\text {seal }}}, B_{\theta D_{\text {seal }}}\right]_{8 \times 14}
$$

$$
\theta=\operatorname{diag}\left(\left[\theta K_{s}, \theta K_{\text {coupling }}, \theta K_{\text {seal }}, \theta D_{\text {seal }}\right]\right)_{14 \times 14}
$$

$B_{\theta}$ and $C_{\theta}$ are constructed as follows and a thorough description of this process can be seen in [29]. It can be shown that changes in stiffness (or damping) in a single direction at e.g. $A_{x}$ corresponds to a change in a single column of system matrix $A$, which corresponds to the node $\mathrm{j}$ where the stiffness (or damping) is altered.

$$
A_{\Delta_{f}}=\left[\begin{array}{ccccccc}
0 & \ldots & 0 & a_{1, j} & 0 & \ldots & 0 \\
0 & \ldots & 0 & a_{2, j} & 0 & \ldots & 0 \\
\vdots & \ddots & \vdots & \vdots & \vdots & \ddots & \vdots \\
0 & \ldots & 0 & a_{i, j} & 0 & \ldots & 0
\end{array}\right]
$$

The change of the system matrix in reduced form $A_{\Delta}$ is found using the same modal truncation matrices as used to reduce the nominal system, as shown in Eq. (11). $A_{\Delta_{f}}$ in Eq. (10) can also be written as a column vector $B_{\theta_{f}}$ and a row vector $C_{\theta_{f}}$ and the change $\theta$. The input mapping $B_{\theta}$ and output mapping $C_{\theta}$ of the uncertainties are thus given as shown in Eq. (13). Repeating this process 14 times (one for each stiffness and damping parameter) and assembling the coloums of $B_{\theta}$ and rows of $C_{\theta}$ and making $\theta$ a $14 \times 14$ diagonal matrix, yields the complete representation.

$$
\begin{aligned}
A_{\Delta} & =T_{L} A_{\Delta_{f}} T_{R} \\
& =T_{L} B_{\theta_{f}} \theta C_{\theta_{f}} T_{R} \\
& =B_{\theta} \theta C_{\theta}
\end{aligned}
$$

\subsection{Estimation of Optimal Parameters}

A current signal $i_{e}$ can be imposed to perturb the system model and a time domain simulation can be employed to yield the displacement response $y$, which can be compared to a response quantified experimentally. To estimate the uncertain AMB parameters the identification scheme is formulated as a minimization problem that iterates through the uncertain parameters to decrease the discrepancy between the simulated response and the response experimentally acquired. The goal is to find the parameters that provide the best fit between simulation data and experimental data. This can be done by finding the global minimum of the cost function shown in Eq. (14) which is defined as the sum of squares of the discrepancy between simulation data and experimental data as

$$
\left.J(\theta)=\| y_{\text {meas }}-y\right) \|_{2}^{2}
$$


Copyright Minimization of the cost function seen in Eq. (14) has been implemented using MATLAB's lsqnonlin function. The method converges fast towards optimal parameters, even for the case where multiple parameters are simultaneously identified.

\subsection{Identification of AMB-rotor Model Parameters}

The first step in obtaining a global model which describes the complete test-facility and is able to separate the seal dynamics, is to update the mathematical model of the AMBrotor system. For this experiment the flexible coupling is unmounted to isolate the dynamic effect of the AMBs only. The FE model of the rotor is considered sufficiently accurate and the uncertain parameters are considered to be the AMB constants $K_{i}$ and $K_{s}$, one for each degree-of-freedom (DOF), i.e. in all $2 \times 4$ parameters. Optimal parameters have been found using the representation of the changed system in Sec. 3.2 and optimization in Sec. 3.3. Two Pseudo-Random Binary Sequence (PRBS) identification signals have been simultaneously applied, one for each actuator direction, resulting in purely parallel displacements of the rotor. The AMB-rotor model shows a good performance, as shown in the time domain in Fig. 8, although the simulated time response shows slightly larger overshoot than the experimental, suggesting the model lacks a bit of damping to precisely represent the experimental response. The model has also been verified in frequency domain by using a linear chirp signal from $0-150 \mathrm{~Hz}$. It is observed that the model with a high level of accuracy represents experimental data up to approximately $100 \mathrm{~Hz}$ in magnitude and phase as shown for both AMB directions in Fig. 9 and 10. Furthermore, some cross coupling dynamics exist, which are plotted separately for the movement at bearing A and bearing B. These would
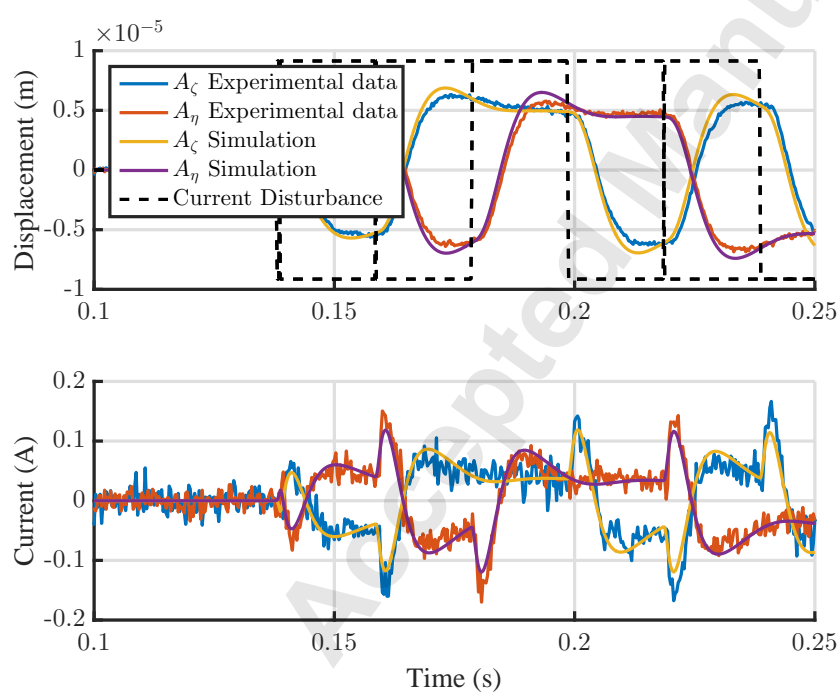

Fig. 8. AMB-rotor model versus experimental time response. Displacements are shown in upper and currents are shown in lower plot. The PRBS current perturbation signal are indicated with dashed lines and the amplitude is scaled to fit the plot. The results are shown for bearing $A$ and are similar for bearing $B$. ideally be zero, however in practice, these could arise from e.g. leakage in the magnetic flux path of the AMBs, the shaft being displaced from magnetic center, sensor measuring uncertainty, neglected base movement etc. The displacements due to cross coupling are generally 10 times smaller when compared to the response in the excitation direction, and they are neglected in the mathematical model. However the cross coupled forces do seem to affect the response in the excitation direction as well, as for example around $30 \mathrm{~Hz}$. The coherence is high from input to output in the excitation direction but lower in the cross coupled directions, probably due to lower displacement amplitude in the cross coupled directions. The correlation matrix in Tab. 2 shows high correlation between $K_{s}$ and $K_{i}$ for each DOF, for example at $A_{\zeta}$, indicating that the dynamic effect of adjusting either $K_{i}$ and $K_{s}$ results in similar effects and their identified values might


Fig. 9. FRFs of AMB-rotor model versus experimental for parallel excitation of rotor in $\zeta$-direction using a $0-150 \mathrm{~Hz}$ chirp signal. The model fits experimental data well up to approximately $100 \mathrm{~Hz}$ indicated with the dashed line. "Simulation" and "Experimental" shows FRFs from simultaneously excitation on bearing $A$ and bearing $B$ to the center movement of the seal in the excitation direction. "Experimental cross, bearing A" and "Experimental cross, bearing B" shows FRFs from excitation on each bearing to the movement in the cross coupled direction - top: amplitude, middle: phase, bottom: coherence 



Fig. 10. FRFs of AMB-rotor model versus experimental for parallel excitation of rotor in $\eta$-direction using a $0-150 \mathrm{~Hz}$ chirp signal. The model fits experimental data well up to approximately $100 \mathrm{~Hz}$ indicated with the dashed line. "Simulation" and "Experimental" shows FRFs from simultaneously excitation on bearing $A$ and bearing $B$ to the center movement of the seal in the excitation direction. "Experimental cross, bearing A" and "Experimental cross, bearing B" shows FRFs from excitation on each bearing to the movement in the cross coupled direction - top: amplitude, middle: phase, bottom: coherence

not be completely unique. $K_{i}$ and $K_{s}$ are theoretically expected to be unique to some degree since $K_{s}$ only affects the bearing stiffness while $K_{i}$ in addition to scaling the stiffness, through the proportional gain of the PID controller, also scales the integration and the derivative gain of the PID controller. Other identification experiments with other bias currents and other parameters of PID gains show that lower correlation between $K_{i}$ and $K_{s}$ can be obtained. Also, the correlation between $K_{s}$ and $K_{i}$ can possibly be lowered significantly by including the measured and simulated current signals in the optimization process. The uniqueness of $K_{i}$ and $K_{s}$ parameters is not considered an issue in this work since the main focus is to obtain an accurate AMB baseline model in order to precisely estimate the seal forces.

\begin{tabular}{|c|c|c|c|c|c|c|c|c|}
\hline & $K_{i, A_{\zeta}}$ & $K_{i, A_{\eta}}$ & $K_{i, B_{\zeta}}$ & $K_{i, B_{\eta}}$ & $K_{s, A_{\zeta}}$ & $K_{s, A_{\eta}}$ & $K_{s, B_{\zeta}}$ & $K_{s, B_{\eta}}$ \\
\hline$K_{i, A_{\zeta}}$ & 1.00 & 0.00 & -0.08 & 0.00 & -0.95 & -0.00 & 0.08 & -0.00 \\
\hline$K_{i, A_{\eta}}$ & 0.00 & 1.00 & 0.00 & -0.08 & -0.00 & -0.96 & -0.00 & 0.08 \\
\hline$K_{i, B_{\zeta}}$ & -0.08 & 0.00 & 1.00 & 0.00 & 0.08 & -0.00 & -0.96 & -0.00 \\
\hline$K_{i, B_{\eta}}$ & 0.00 & -0.08 & 0.00 & 1.00 & -0.00 & 0.07 & -0.00 & -0.96 \\
\hline$K_{s, A_{\zeta}}$ & -0.95 & -0.00 & 0.08 & -0.00 & 1.00 & 0.00 & -0.09 & 0.00 \\
\hline$K_{s, A_{\eta}}$ & -0.00 & -0.96 & -0.00 & 0.07 & 0.00 & 1.00 & 0.00 & -0.09 \\
\hline$K_{s, B_{\zeta}}$ & 0.08 & -0.00 & -0.96 & -0.00 & -0.09 & 0.00 & 1.00 & 0.00 \\
\hline$K_{s, B_{\eta}}$ & -0.00 & 0.08 & -0.00 & -0.96 & 0.00 & -0.09 & 0.00 & 1.00 \\
\hline
\end{tabular}

Table 2. Correlation matrix - calculated based on the Jacobian matrix. Values close to 1 or -1 indicates strong correlation between parameters.

\subsection{Identification of Flexible Coupling Dynamics}

The flexible coupling is modelled as a direct stiffness in each actuator direction, which is identified in a separate experiment where the estimated AMB parameters from previously experiment are kept fixed in order to obtain a unique solution. Again, two PRBS identification signals have been simultaneously applied, one for each actuator direction, resulting in purely parallel displacements of the rotor. The stiffness coefficients of flexible coupling are extracted and the results are shown in Table 3

Table 3. Identified stiffness coefficients of flexible coupling.

$$
\begin{array}{ll}
K_{c_{\zeta}} & 0.40 \times 10^{6} \mathrm{~N} / \mathrm{m} \\
K_{c_{\eta}} & 0.42 \times 10^{6} \mathrm{~N} / \mathrm{m}
\end{array}
$$

\subsubsection{Identification of Seal Coefficients for Various Fre- quencies}

The seal forces are identified with parallel movements of the rotor. This is to avoid axial forces being generated in the discharge system of the seal house. A stepped sine procedure in the range $5-200 \mathrm{~Hz}$ with steps of $10 \mathrm{~Hz}$ is applied as an excitation current signal to obtain seal force coefficients for different excitation frequencies - the same excitation signal that is used for extracting seal coefficients using CFD simulation as shown in [10]. The seal force coefficients for each excitation frequency are found using the representation of the changed system in Sec. 3.2 and optimization in Sec. 3.3. An example of the fit of seal force coefficients identified at $40 \mathrm{~Hz}$ excitation is shown in Fig. 11. Good agreement between the global model and experimental data is found, indicating the seal model structure and the found seal parameters describes the actual seal forces well. The cross coupled forces of the seal are clearly seen since the excitation is applied in the $\zeta$-direction while a large response is also seen in the $\eta$-direction.

The stepped sine identification procedure has been repeated for differential pressures across the seal of $0.7 \mathrm{bar}$, 



Fig. 11. Example of the fit of seal coefficients identified at $40 \mathrm{~Hz}$ excitation. The simulated and experimental time response are shown for displacement (upper) and for current (lower). A sinus excitation current is applied in the $\zeta$-direction.

$1.0 \mathrm{bar}$ and $1.2 \mathrm{bar}$ to investigate the influence of different pressures. The results, in terms of seal coefficients for different pressures across the seal, are shown in Fig. 12. As a reference, the identified seal coefficients, for when no pressure is applied, are plotted along with the other results. These reference coefficients indicates how biased the other coefficients might be due to residual dynamics that might not have been captured by the AMB-rotor-coupling model, such as the cross coupled effects of the AMB-rotor model. The following is observed from Fig. 12:

1. The direct stiffness values $K$ around zero are expected since the seal clearance, simulating a worn seal, is large.

2. The cross coupled stiffness values $k$ are large and change significantly with the pressure across the seal. This parameter does significantly effect the dynamic of the overall system. It is expected that the cross coupled stiffness is large due to high preswirl in the seal. Moreover $k$ seems to be frequency-independent as also found using bulkflow modeling for annular gas seals in [30].

3 . The direct damping $D$ decreases as the excitation frequency increases, however; this tendency must be disregarded since the tendency is also captured by coefficients found for no pressure. The direct damping is low and seems to depend on the applied pressure.

4. The cross coupled damping $d$ is low and depends on the applied pressure. High pressure yields high cross coupled damping.

The uniqueness of the found seal coefficients is high, since all parameters are very weakly correlated, as shown in Table 4.

\subsubsection{Verification of Global Model in Frequency Dom- ain Including Fixed Frequency Seal Models}

To verify the performance of the global model and the frequency independence of the identified test seal, a comparison is made between the frequency response functions of the simulated model responses with the experimental responses obtained from test-facility for different pressures. The global model includes a seal model with constant coefficients. These coefficients are highlighted as solid lines shown in Fig. 12. Datasets from the identifications of seal coefficients for different frequencies in Sec. 3.5.1 have been reused for the verification. The global model fits well up to approximately $100 \mathrm{~Hz}$, which is the frequency range in which the AMBrotor model is considered valid. This is shown for pressures 0.7 bar, 1.0 bar and 1.2 bar in Fig. 13, 14 and 15. Hence, the test seal is practically independent by frequency since the FRF can be matched even if constant values for stiffness and damping are assumed.

It is interesting that the frequency plot in Fig. 13 shows no significant resonance peak since the control system is able to suppress the rigid mode shapes of the system. However, as the pressure increases to 1.2 bar and the cross coupled stiffness increases, a resonance appears, as shown in Fig. 15. This indicates robustness and stability issues start to arise since the direct stiffness of the AMBs with their current controller configuration is not large enough for the cross coupled effects to be neglectable. Hence, to identify seal forces with higher pressures, the controller must either yield a larger direct stiffness or be designed to counteract the cross-coupled forces arising from the seal.

\section{Conclusion}

This paper demonstrates an elegant way of identifying the uncertain or unknown parameters with a focus on dynamic seal forces in rotordynamic AMB systems, step by step, without changing the system order of the global model. This is done by utilizing the structure of the model, the shaft model and closed loop system identification. This procedure is applied to a rotordynamic test-facility to identify the uncertain parameters of the dynamic AMB model, the flexible coupling forces and dynamic annular seal forces.

The identified seal coefficients of the tested seal with gas fluid shows practically no excitation frequency dependence.

\begin{tabular}{|c|c|c|c|c|}
\hline & $\mathrm{K}$ & $\mathrm{D}$ & $\mathrm{k}$ & $\mathrm{d}$ \\
\hline $\mathrm{K}$ & 1.00 & -0.00 & 0.01 & -0.04 \\
\hline $\mathrm{D}$ & -0.00 & 1.00 & 0.04 & 0.01 \\
\hline $\mathrm{k}$ & 0.01 & 0.04 & 1.00 & -0.00 \\
\hline $\mathrm{d}$ & -0.04 & 0.01 & -0.00 & 1.00 \\
\hline
\end{tabular}

Table 4. Correlation matrix - calculated based on the Jacobian matrix. Values close to 1 or -1 indicates strong correlation between parameters. The values are shown for identification of seal coefficients with $40 \mathrm{~Hz}$ excitation frequency. 


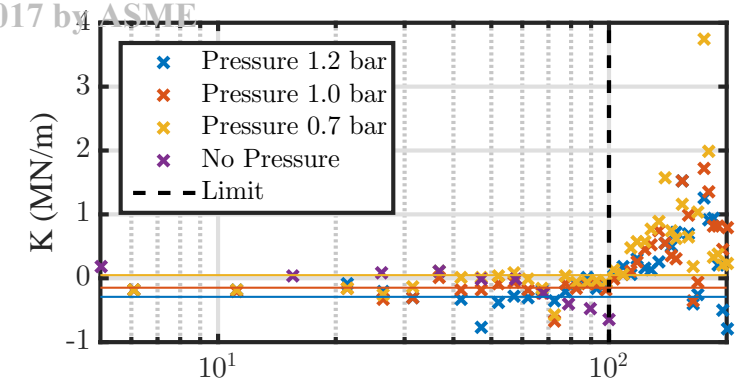

Freq. (Hz)

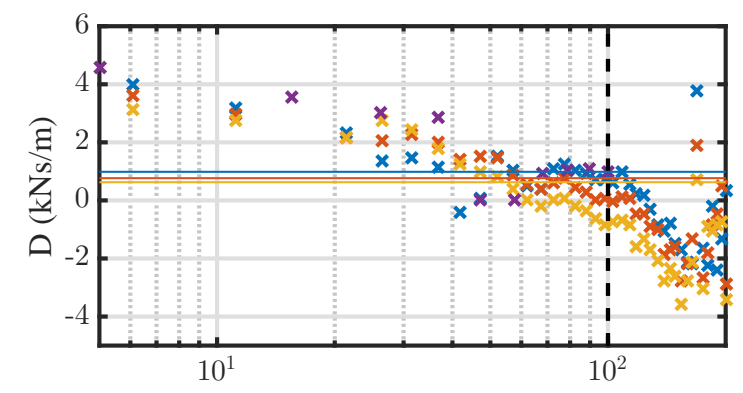

Freq. $(\mathrm{Hz})$

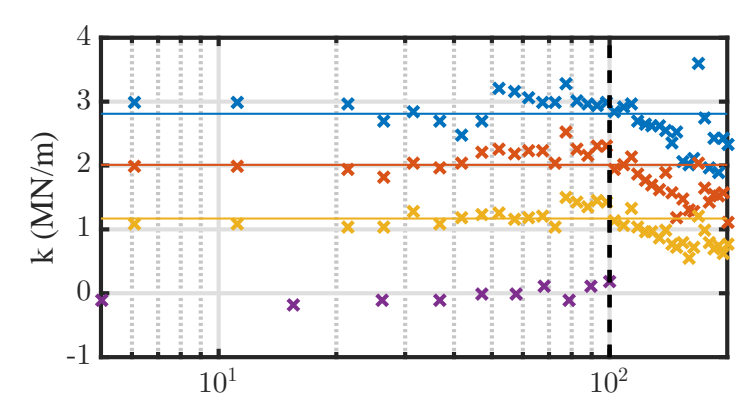

Freq. (Hz)

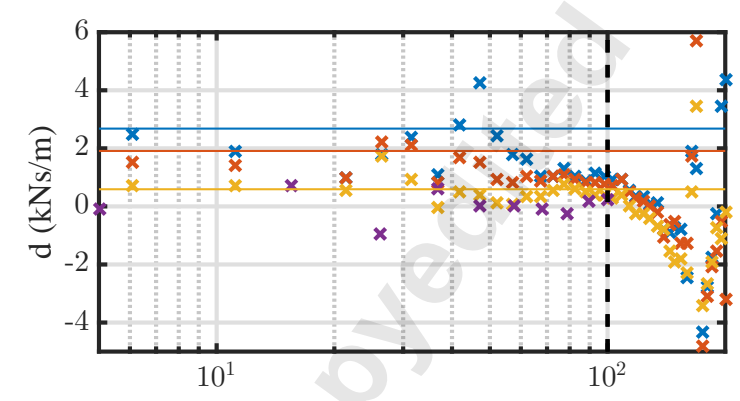

Freq. $(\mathrm{Hz})$

Fig. 12. Seal coefficients vs. excitation frequencies for different pressures across the seal. Coefficients are valid up to approximately $100 \mathrm{~Hz}$ as indicated with the dashed line. The solid lines indicates the constant seal coefficients chosen for model verification in Sec. 3.5.2.

This fits well with previous results found using bulkflow modeling of annular seals using gas fluid in [30]. The most significant seal parameter is the cross-coupled stiffness which is highly dependent on the pressure across the seal. The test seal furthermore seems to yield some cross coupled damping. The direct stiffness and direct damping coefficients of the test seal are practically zero.

Precise identification of seal parameters, using the presented approach, relies on an accurate mathematical baseline model of the AMB-rotor-coupling system. However, this paper demonstrates that it is possible to obtain an indication of uncertainties and erroneous tendencies caused by imperfections of the mathematical baseline model. An erroneous tendency that should be disregarded is shown for the direct damping.

The excitation frequency-independence of the test seal is verified using a set of fixed seal coefficients to build a global model which fits experimental data well. This model is thus only dependent on the pressure across the seal.

The identification approach is applied to a test rig where only one seal in back-to-back configuration is present. In reality a turbomachinery will have multiple seals at several locations and each of them will affect the lateral rotordynamic response. The approach might also be applied to industrial machines equipped with multiple seals, but with caution. In case of one or two seal locations, the approach will still lead to the characterization of dynamic effect of each individual seal at its specific location. Nevertheless, in case of machines equipped with multiple seals at more than two locations, equivalent values of force coefficients of multiple seals are expected, and the characterization of dynamic effect of each individual seal will hardly be achieved. It is also important to mention that the approach is aimed to machines in which the changes of seal dynamic forces happen relatively slow. For sudden changes of seal forces in short period of time, the approach needs to be revised and expanded.

\section{References}

[1] Childs, D. W., 1993. Turbomachinery rotordynamics: phenomena, modeling, and analysis. John Wiley \& Sons.

[2] Picardo, A., and Childs, D. W., 2005. "Rotordynamic coefficients for a tooth-on-stator labyrinth seal at 70 bar supply pressures: measurements versus theory and comparisons to a hole-pattern stator seal". Journal of engineering for gas turbines and power, 127(4), pp. 843-855.

[3] Soulas, T., and San Andres, L., 2007. "A bulk flow model for off-centered honeycomb gas seals". Journal of Engineering for Gas Turbines and Power, 129(1), pp. 185-194.

[4] Scharrer, J. K., 1988. "Theory versus experiment for the rotordynamic coefficients of labyrinth gas seals: Part ia two control volume model". Journal of Vibration, Acoustics, Stress, and Reliability in Design, 110(3), pp. 270-280.

[5] Iwatsubo, T., 1980. "Evaluation of instability forces of labyrinth seals in turbines or compressors".

[6] Millsaps, K. T., and Martinez-Sanchez, M., 1994. "Dynamic forces from single gland labyrinth seals: Part i) ideal and viscous decomposition". Journal of turbomachinery, 116(4), pp. 686-693.

[7] Childs, D. W., and Vance, J. M., 1997. "Annular gas se- 

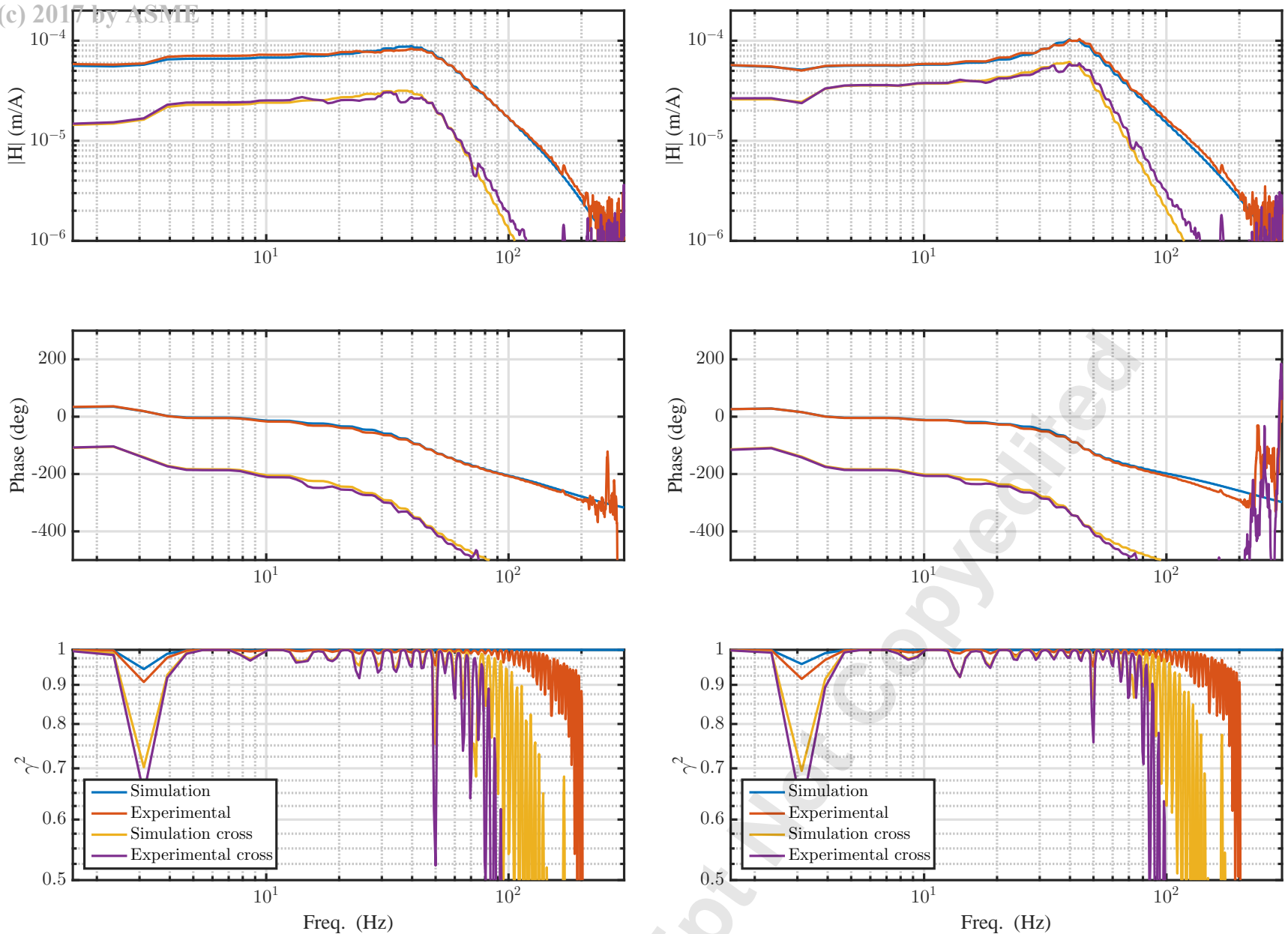

Fig. 13. FRFs of global model with constant seal coefficients versus experimental using 0.7 bar pressure across the seal - top: amplitude, middle: phase, bottom: coherence

als and rotordynamics of compressors and turbines". In Proceedings of the 26th Turbomachinery Symposium, pp. 201-220.

[8] Seifert, B. A., 2007. "Measurements versus predictions for rotordynamic coefficients and leakage rates for a novel hole-pattern gas seal”. PhD thesis, Texas A\&M University.

[9] Vannarsdall, M. L., 2012. "Measured results for a new hole-pattern annular gas seal incorporating larger diameter holes, comparisons to results for a traditional hole-pattern seal and predictions". $\mathrm{PhD}$ thesis, Texas A\&M University.

[10] Nielsen, K. K., Jonck, K., and Underbakke, H., 2012. "Hole-pattern and honeycomb seal rotordynamic forces: Validation of cfd-based prediction techniques". Journal of Engineering for Gas Turbines and Power, 134(12), p. 122505.

[11] Noronha, R. F. d., Miranda, M. A., Lucchesi-Cavalca, K., Memmott, E. A., Ramesh, K., et al., 2014. "Stability testing of co2 compressors". In Proceedings of the 43rd Turbomachinery Symposium, Texas A\&M University.



Fig. 14. FRFs of global model with constant seal coefficients versus experimental using 1.0 bar pressure across the seal - top: amplitude, middle: phase, bottom: coherence

[12] Wagner, N. G., Steff, K., Gausmann, R., and Schmidt, M., 2009. "Investigations on the dynamic coefficients of impeller eye labyrinth seals". In Proceedings of the Thirty-Eighth Turbomachinery Symposium, Houston, TX, September, pp. 14-17.

[13] Kocur, J. A., Nicholas, J. C., and Lee, C. C., 2007. "Surveying tilting pad journal bearing and gas labyrinth seal coefficients and their effect on rotor stability". In 36th Turbomachinery Symposium, Turbomachinery Laboratory, Texas A\&M University, College Station, TX, September, pp. 10-13.

[14] Ertas, B. H., Delgado, A., and Vannini, G., 2012. "Rotordynamic force coefficients for three types of annular gas seals with inlet preswirl and high differential pressure ratio". Journal of Engineering for Gas Turbines and Power, 134(4), p. 042503.

[15] Elrod, D., Nicks, C., Childs, D., and Nelson, C., 1985. "A comparison of experimental and theoretical results for rotordynamic coefficients of four annular gas seals". Progress Report NASA contract NAS8-33716 Texas A\&M University, Turbomachinery Laboratories, Mechanical Engineering Department College Station, 

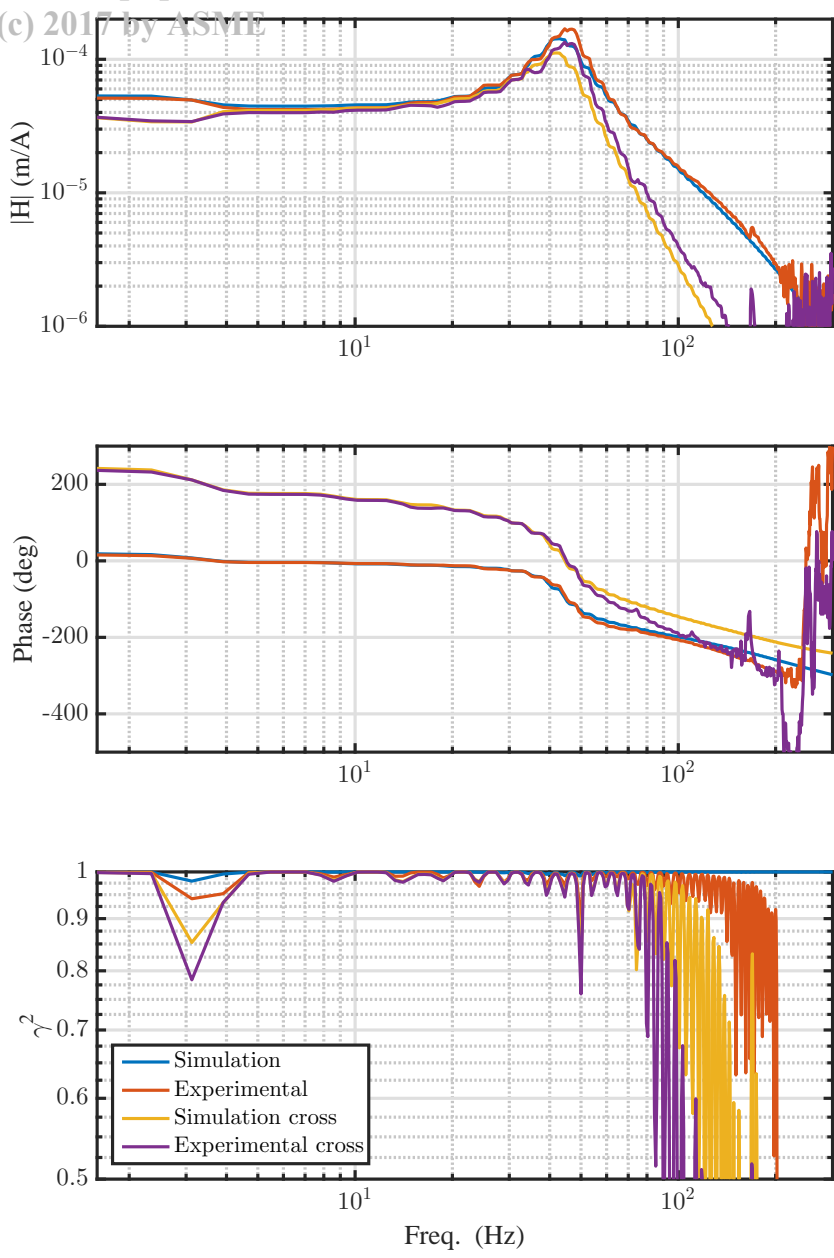

Fig. 15. FRFs of global model with constant seal coefficients versus experimental using 1.2 bar pressure across the seal - top: amplitude, middle: phase, bottom: coherence

\section{Texas, $\mathbf{7 7 8 4 3 .}$}

[16] Brown, P. D., and Childs, D. W., 2012. "Measurement versus predictions of rotordynamic coefficients of a hole-pattern gas seal with negative preswirl". Journal of Engineering for Gas Turbines and Power, 134(12), p. 122503.

[17] Wagner, N., and Steff, K., 1997. "Dynamic labyrinth coefficients from a high-pressure full-scale test rig using magnetic bearings". Demag A. G, Rotordynamic Instability Problems in High-Performance Turbomachinery (SEE N 97-24525 01-37), pp. 95-111.

[18] Knopf, E., and Nordmann, R., 1998. "Active magnetic bearings for the identification of dynamic characteristics of fluid bearings". In Proceedings 6th International Symposium on Magnetic Bearings, Cambridge, MA, August, pp. 5-7.

[19] Matros, M., and Nordmann, R., 1997. "Dynamic characteristics of a hydrostatic bearing identified by active magnetic bearings". Kaiserslautern Univ, Rotordynamic Instability Problems in High-Performance Turbomachinery 1996 p 23-28(SEE N 97-24525 01-37).

[20] Nordmann, R., and Aenis, M., 2004. "Fault diagno- sis in a centrifugal pump using active magnetic bearings". International Journal of Rotating Machinery, 10(3), pp. 183-191.

[21] Aenis, M., and Nordmann, R., 2002. "Fault diagnosis in rotating machinery using active magnetic bearings". In 8th International Symposium on Magnetic Bearing, p. 125.

[22] Zutavern, Z. S., and Childs, D. W., 2008. "Identification of rotordynamic forces in a flexible rotor system using magnetic bearings". Journal of Engineering for Gas Turbines and Power, 130(2), p. 022504.

[23] Voigt, A. J., 2016. Towards Identification of Rotordynamic Properties for Seals in Multiphase Flow Using Active Magnetic Bearings. Design and Commissioning of a Novel Test Facility. Technical University of Denmark.

[24] Voigt, A. J., Mandrup-Poulsen, C., Nielsen, K. K., and Santos, I. F., 2017. "Design and calibration of a full scale active magnetic bearing based test facility for investigating rotordynamic properties of turbomachinery seals in multiphase flow". Journal of Engineering for Gas Turbines and Power, 139(5), p. 052505.

[25] Lauridsen, J. S., and Santos, I. F., 2016. "Design of robust amb controllers for rotors subjected to varying and uncertain seal forces".

[26] Bleuler, H., Cole, M., Keogh, P., Larsonneur, R., Maslen, E., Okada, Y., Schweitzer, G., Traxler, A., Schweitzer, G., Maslen, E. H., et al., 2009. Magnetic bearings: theory, design, and application to rotating machinery. Springer Science \& Business Media.

[27] Nelson, H., 1980. "A finite rotating shaft element using timoshenko beam theory". Journal of mechanical design, 102(4), pp. 793-803.

[28] Skogestad, S., and Postlethwaite, I., 2007. Multivariable feedback control: analysis and design, Vol. 2. Wiley New York.

[29] Lauridsen, J. S., Sekunda, A. K., Santos, I. F., and Niemann, H., 2015. "Identifying parameters in active magnetic bearing system using lft formulation and youla factorization". In 2015 IEEE Conference on Control Applications (CCA), IEEE, pp. 430-435.

[30] San Andrés, L., 2012. "Rotordynamic force coefficients of bubbly mixture annular pressure seals". Journal of Engineering for Gas Turbines and Power, 134(2), p. 022503. 